\title{
3D TERRESTRIAL LASER TECHNOLOGY IN SPORTING CRAFT 3D MODELLING
}

\author{
Arias, P.; Armesto, J.; Lorenzo, H. \& Ordóñez, C. \\ Department of Natural Resources and Environmental Engineering, Universidad de Vigo, \\ E.T.S.I. MINAS, Campus Universitario, 36310 Vigo, Spain \\ E-Mail: cgalan@uvigo.es
}

\begin{abstract}
The urgent need to increase productivity and competitiveness demands from sporting craft builders the incorporation of newer design and manufacturing technologies, as CAD/CAM and CNC machining systems. This paper describes the sporting boats' 3D surface modelling, needed for automated manufacturing processes, throug terrestrial laser scanner technology. The methodology followed for data collection and data processiong is described in detail, advantages and limitations of this technique are displayed and the accuraccy of the obtained results is also estimated. According to obtained results, this technology proves to provide accurate results and to be cost and time effective.

(Extended paper from the International Symposium CompIMAGE - Coimbra, Portugal, 20-21 October 2006.)
\end{abstract}

Key Words: Sporting Craft, Terrestrial Laser Scanner, 3D Modelling

\section{INTRODUCTION}

The demand for fishing, sporting and pleasure craft in developed countries has undergone a steady increase in recent years and all medium-term forecasts point towards the continuity of this trend, due to growing purchasing power and greater leisure time of citizens in these countries. This fact, together with the gradual decrease within the European shipping market [1], are the likely causes of the increasing number of companies entering the sporting boats sector.

Nowadays, most sporting boat manufacturing processes are largely manual and rely on the expertise of individual operatives; resulting boats are therefore usually unique models and differ from any other. In addition, rigorous quality control programmes and assembly flow diagrams are rarely implemented or applied during their construction. As a result, parts are frequently wasted or re-worked due to production errors, causing production delays and cost increases. This situation combined with the urgent need to increase productivity and competitively, is placing pressure on shipbuilders to improve production processes by incorporating new design and manufacturing technologies. Automation is one major step towards achieving the quality, cost and delivery targets and the coordination of design and manufacturing processes.

Due cost effectiveness and high quality results, CAD/CAM and CNC machining is one of the largest areas of expansion and renovation in the automation and coordination of design and manufacturing processes in a wide range of manufacturing processes [2]. In order to program the machine's operations, this manufacturing process requires 3D digital models of the objects that are to be manufactured. Reverse engineering, which concerns with the generation of 3D CAD models of physical objects, may be employed to obtain digital models of boats in situations wherein the serial automated replication of an existing boat model is needed (because of satisfactory aesthetic design and successful navigability performance).

Apart from its usefulness in the automation of manufacturing, having a 3D digital model of the product is vital for the following concerns: a) the reuse of the information for reengineering or repair processes (when analysis and modifications are required to construct a 
new and improved product); b) the navigability simulation field; c) the product documentation for virtual advertising and merchandising systems; d) the possibility of sending an electronic format of the model to allow the replication of the boat in different, remote sites [3]; e) quality control processes (through shape plans, symmetry analysis, etc).

Terrestrial laser scanner is a recently developed reverse engineering technology for data collection and subsequent 3D modelling whose applications in industrial and civil construction fields are increasing day by day. The outlines for effective terrestrial laser scanning in capturing point clouds and measuring distances are described in [4]. According to [5] laser scanning technology might be considered valid and precise for creating high-resolution polygon meshes, surfaces or solid models of objects. Nevertheless achieving smooth surface representations (needed for reasons of manufacturability) from point clouds is the key task for the efficient application of this technology to reverse engineering processes [6].

The problem of obtaining smooth surface models from laser point clouds can be stated as follows: given a set $V$ of $n$ points in the 3D space, a surface $S$ might be found that approximates or interpolates $V$. A huge variety of procedures have been developed in the last years concerning this task. When unstructured data are focused, generally a first triangulation of the points is performed, in other words, a piecewise linear function is obtained that interpolates $V$. This introduces a first level of organization as it defines the basic topological structure of the point cloud. The process of remeshing to obtain a regular polygon mesh can be considered a second level of organization that leads to a better smooth surface approximation [7]. The next step is segmentation into smaller point regions, in such a way that later each region can be well-aproximated by a single surface. Finally the individual surfaces are trimmed and joined together [8].

For the segmentation three main options might be accomplished: iterative approaches or user assistance for segmentation or holding strong assumptions related to the object topology [8]. Automatic segmentation procedures that have been developed until the date, besides to be sensitive or computationally complex or both, can only be applied to simple topology data [9]; consequently they are feasible in a limited number of applications since several industrial environments, as boats structure, can not be represented as a set of plans, cylinders, spheres, and other simple surfaces.

In this paper a boat 3D modelling procedure from unstructured laser scanned data is described. It is based on an automated polygonation, user guided segmentation and semiautomated surface fitting. Advantages and limitations of this technique are displayed and the accuracy of the obtained results is also estimated.

This paper is a part of a broader project whose final aim consists of building a pleasure boat prototype with reinforced polyester with fibreglass on the basis of the structure of an existing one. The 3D digital models of the deck and the hull are needed to carry out reengineering and redesigning processes, navigability simulations, etc. The size of the existing boat measures $12 \mathrm{~m}$ in overall length, with a beam of $4.5 \mathrm{~m}$ and the draft measuring $2.4 \mathrm{~m}$.

\section{INSTRUMENTATION}

The equipment required to implement the proposed methodology is listed as follows:

- Terrestrial laser scanner: A Leica Cyrax 2500 laser was used. This is a pulsed time-offlight scanner that records up to 1000 points/second with a precision of $6 \mathrm{~mm}$ with a resolution capacity of less than $1 \mathrm{~mm}$ [10]. For maximum distances up to 50 metres the precision achieved is around $2 \mathrm{~mm}$. Each scanning operation requires approximately 10 minutes and each scan covers a $40^{\circ}$ vertical x $40^{\circ}$ horizontal field-of-view. 
- Magnetic targets. Automatically recognised by the software and used as control points.

- Laptop computer: Pentium IV, 2.4 GHz, 1 GB RAM, 40 GB hard disk. Terrestrial laser applications require the scanner to be connected to a computer in which the point clusters recorded by the laser are stored in real time.

- Tripod. A tripod provides the necessary support to ensure the terrestrial laser scanner is set at suitable height and kept stable and immobile during scanning operations.

- Software application for point cloud matching.

- Software for point deleting and point cloud filtering.

- Software for generating 3D surface models from the pre-processed point clouds.

- Software for creating hull and deck cross-sections from the 3D surface models.

Resulting models are based on standard file formats (e.g. IGES) compatible with most of the commercial software.

\section{METHOD}

In this section we describe the methodology employed to obtain an accurate threedimensional model of the deck and hull of a prototype pleasure fishing boat. This methodology is performed in five sequential stages which are summed up next. First two stages correspond to field work and the last three to laboratory work.

\subsection{Data collection design}

This step focuses on the design of the data collection phase in order to optimize reflectivity conditions and to minimize measuring time and distorting factors during data capture. The following points need to be borne in mind:

- Idenfication of suitable positions from which to capture data using the laser, avoiding shadow areas and maintaining overlap between consecutive scans, whilst keeping the number of scans to a minimum.

- Environmental factors. Surrounding environment is analyzed in order to detect those elements in or around the scanning area that could interfere in data collection: sources of vibrations, shadowing elements, etc.

- Craft support. The stability of the craft support is verified in order to avoid any kind of vibrations or movement during the scanning process.

- Correct lighting conditions. This is an essential parameter, as the point clouds assembly is based on magnetic target recognition, and they can only be recognised when light is diffused and at the right intense.

\subsection{Data collection}

During the scanning process, the following guidelines were followed:

- Magnetic target distribution. Magnetic targets were employed because they are automatically recognised by the software system, so subsequent assembly of point clouds is faster and easier. Ten magnetic targets were distributed over and around the craft, in such a way that at least five were registered with each scan.

- Scanner stationing and data collection. Any vibration, strong noise or any movement of the scanner or the craft itself are avoided during the scanning process.

- Point cloud assembly. Each registered cloud of points was added to the others after scanning in order to detect incomplete data, corrupted files, etc. Prior to assembly, magnetic targets were identified and marked in each point cloud. Later these magnetic targets were taken as a reference for point clouds matching. At the end of this phase all the collected point clouds were aligned in a common global coordinates system. 


\subsection{Data pre-processing}

Once the fieldwork phase is completed, the next stage is data pre-processing. This stage is oriented to delete points that will not be neccesary to construct the 3D surface model in the final stage. Deck and hull data were processed independently of each other.

Pre-processing is structured as follows:

- Point deletion. Due to field of vision of the scanner, several elements apart from the craft were recorded: walls, ceiling and floors of the premise, other boats, work tools and other materials, etc. In this stage the scans are analysed in order to detect and remove points that do not belong to the objects that are to be modelled. Moreover, points that were registered with erroneous values and that are clearly outside the area of the craft surface were deleted. The $52 \%$ of the entire hull assembled point cloud and the $66 \%$ of the deck point cloud were deleted in this step. The resulting point cloud of the hull is shown in Fig. 1.

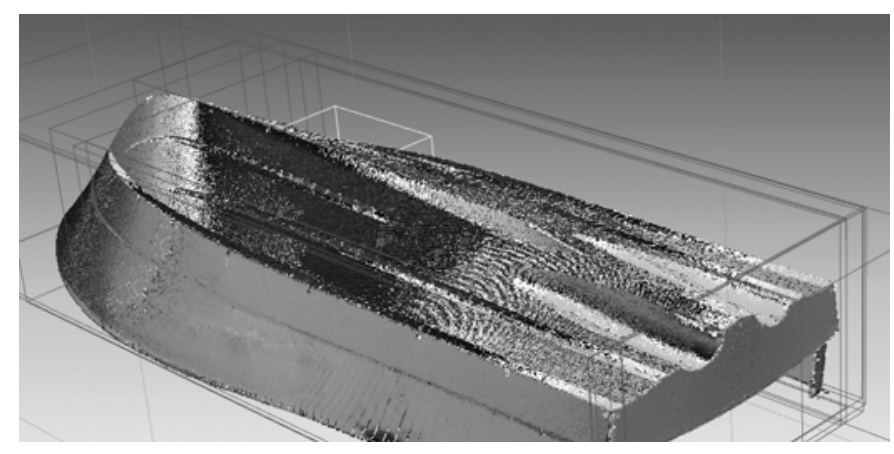

Figure 1: Assembled point clouds for the craft hull after deleting points.

- Redundancy filtering. As a consequence of the data capture design (overlap between different scans is needed in order to reassemble), there is a significant amount of redundant information in the assembled point cloud. In this phase this redundant information was removed from the cloud of points in order to reduce the volume of data and to simplify subsequent operations (see Fig. 2 for resulting hull point cloud). The filtering intensity is reliant on the available hardware and software for data processing. Nevertheless, filtering might be light in areas with complex curves and surfaces. It can be more intense in areas with simple shapes, as lots of middling points in these areas do not show any relevant information for the construction of the 3D models. Filtering is performed through deletion of points whose distance with others is within a previously established global mean distance. Only the $6 \%$ of the entire hull point cloud left after redundancy filtering. For the deck the $19 \%$ was preserved.

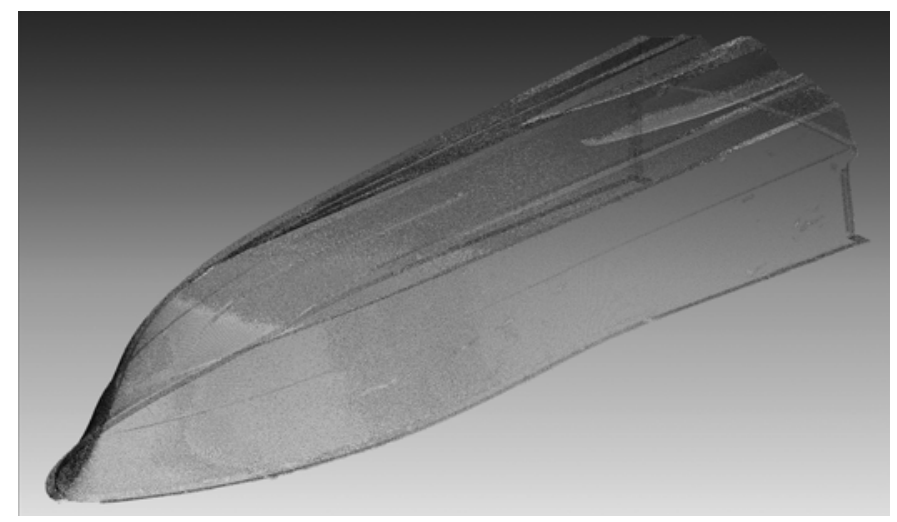

Figure 2: Assembled point clouds for the craft hull after redundancy filtering. 


\subsection{Data processing: craft 3D modelling}

The 3D polygon mesh models were constructed either for the hull (see Fig. 3) and the deck of the craft from the filtered point clouds. The same procedures were performed for the hull and the deck.

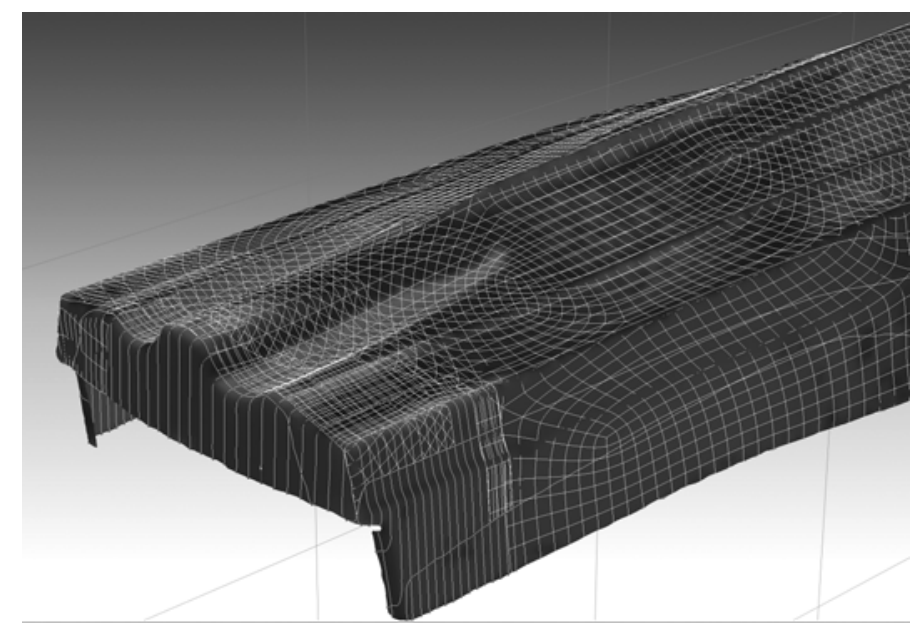

Figure 3: Detail view of the hull polygon mesh model.

The hull modelling was performed as follows:

- Autosurfacing. This is a semi-automated process where NURBS surfaces (Non-Uniform Rational B-Spline) are fitted to the polygon mesh. Primary, user guided segmentation is performed. Boundaries of regions are selected from the polygon mesh lines. Then, the number of surfaces needed for the description of the topology of the craft is optimized automatically through decimation of the initial polygon mesh. For each region, a NURBS surface is fitted. Resulting surfaces are joined to obtain the whole model. Some joins and edges are manually smoothed in a stronger or lighter degree depending on the kind of surface and the characteristics of the element to be modelled.

- Identification of incomplete surfaces and manual completion. Those areas where the system was unable to automatically generate the corresponding surfaces were manually completed until the whole three-dimensional model of the craft was obtained (see Fig. 4 for result).

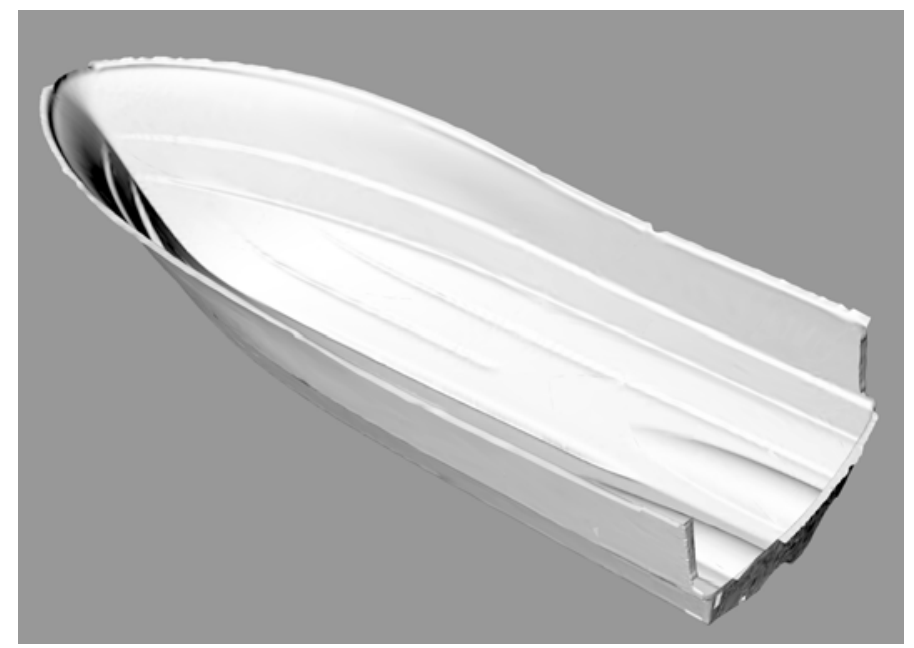

Figure 4: Resulting hull surface 3D model. 
Autosurfacing could not be performed for the deck modelling due to the abundance of elements withcomplex curves and surfaces. In this case it was performed as follows:

- Sectioning. The polygon mesh model of the craft deck was sectioned into a series of transversal and longitudinal planes, obtaining a set of section slides. For each section slide appropriate curves where derived: straight lines, regular curves and NURBS were used. Fig. 5 shows a central cross-section of the boat, located at a distance of $675 \mathrm{~mm}$ from the bow, where the asymmetry was up to $30 \mathrm{~mm}$ in the upper part of the hull, i.e. the part nearest the deck.

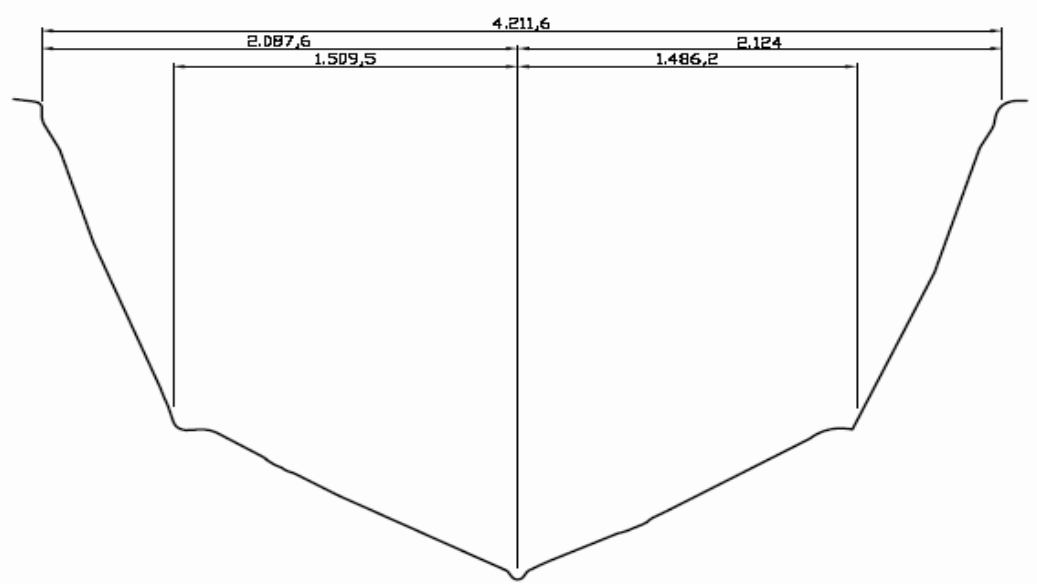

Figure 5: Section of the hull showing the stern tubes where there are not significant asymmetries.

- Surface generation. The transversal and longitudinal planes defined in the previous step divide the deck into sections. The corresponding surface was obtained for each section taking its boundary edges (resulting of the previous step) as reference and defining backup rail edges when polysurfaces (considered as surfaces formed of NURBS [11]) should be fitted to the polygon mesh model of the deck.

\subsection{Accuracy assessment of the modelling process}

No method for laser data accuracy analysis has yet emerged that is generally accepted [12], [13]. The accuracy of the resulting 3D models is especially difficult to estimate when the real shape of the object is unknown and the measuring equipment has not been previously calibrated. As these should be the expected circumstances in most cases where laser scanning would be applied to boat modelling, the thickness of the non filtered point clouds have been considered the most adequate indicator of the quality of the data collection process and the point positioning accuracy. To assess the thickness of the point clouds, the scans aligned in a global coordinates system are sectioned by perpendicular planes to the stern-bow symmetry axis. Twenty homogeneously distributed sections were analyzed.

\section{RESULTS}

Data collection of the targeted boat with Leica laser scanning needed eight scans: 4 for the hull, 4 for the deck; these were taken from seven different stations. All the scans were registered in the computer using the software CYCLONE V.4.0 (Leica Geosystems) and the results were satisfactory: the effect of the alignment errors (the misclosure obtained when considering all the scans together) was $5 \mathrm{~mm}$ in standard deviation and the cloud noise standard deviation (the average of the standard deviations obtained for each pair of scans at the overlapped areas) $6 \mathrm{~mm}$. 
Over six million points were measured, but only the $6 \%$ and $19 \%$ of the point clouds of the hull and deck respectively (see Table I for quantitative details) were processed for the polygon mesh generation. It has been observed that laser data processing requires experienced and skilful operators, especially for redundancy filtering step, as there is a risk of filtering out too much data, with the consequent loss of information, or too little data filtering, causing subsequent problems due to excessive information and overly large files.

The time needed either for data collection or for each step of data processing and 3D modelling is indicated in Table I as a percent value of the whole time taken up by the whole process.

Note that though a great amount of points is measured, data collection is the less time consuming step ( $8 \%$; see Table I), while completing surfaces consumes most processing time. In this last step strong differences between hull and deck modelling appear. The craft hull, which is a surface with few frequently smooth discontinuities, was automatically modelled by autosurfacing in the $80 \%$ of its surface. On the other side, autosurfacing could not be performed for the deck modelling, mainly due to plenty of features with different sizes and shapes, and especially of parts with complex curves and surfaces. In this case regular and irregular curves were obtained for a set of 120 section slides of the deck polygon mesh, and then the corresponding surfaces could be obtained. This step required the $70 \%$ of the whole time (see Table I).

According to these results, terrestrial laser scanning might be considered a cost and time efficient technology for craft hull 3D modelling. Moreover, although the deck modelling has proved to be a slow hard manual process, terrestrial laser scanning should be considered a competitive technology for deck craft 3D modelling, in relation to traditional modelling techniques, either manual or topographic methods.

The precision analysis of the modelling process data through Leica terrestrial laser scanner, estimated by the thickness of the point clouds, showed that accuracy values decrease in proportion to the distance between the laser and the object being measured. In this case it was confirmed that thickness values are less than $5 \mathrm{~mm}$ for the analyzed sections of the boat.

Table I: Quantitative details of a sporting boat 3D modelling process (deck and hull separately) through terrestrial laser scanner. The time consumes of each step is indicated as a percentage value of the whole processing time.

\begin{tabular}{|c|c|c|c|c|}
\hline Step & Hull & Time (\%) & Deck & Гime (\%) \\
\hline Data collection & $2,454,784$ points & 4 & 1,178,197 points & 4 \\
\hline Points deleting & $1,169,237$ points & 3 & 520,952 points & 3 \\
\hline Redundancy filtering & 141,841 points & 2 & 217,976 points & 2 \\
\hline Polygon mesh model & 282,600 faces & 2 & 469,950 faces & 2 \\
\hline Autosurfacing / sectioning & 92 surfaces & 1 & 3,424 section slides & 2 \\
\hline Manual completion / surfaces generation & 24 surfaces & 6 & $\begin{array}{l}277 \text { polysurfaces, } \\
95 \text { regular surfaces }\end{array}$ & s 70 \\
\hline
\end{tabular}

Although the positioning accuracy of points measured through laser scanner technology is highly dependent on signal reflected from the surface, and this factor itself depends on varying parameters such as the colour and the roughness of the surface, or the angle at which the surface is scanned, this maximum value of thickness might serve as a reference for the accuracy to expect when sporting boats with similar size are measured. 


\section{CONCLUSIONS}

The construction of pleasure and fishing boats to high standards of quality requires current manufacturing methods based on manual skill and ad hoc modifications to be replaced by more accurate automated methods. One possibility is to apply reverse engineering techniques to the manufacturing process. This would ensure accurate validation of the product at the design stage and eliminate re-work at the construction stage.

This study clearly shows the reliability of terrestrial laser scanning in the generation 3D surface models of pleasure craft. These models, as well as being essential for automated production processes, also allows for engineering processes, adjustments to be made to the deck and hull, navigability simulations, quality control concerning asymmetry etc. Many of these processes cannot be carried out using traditional measuring techniques (tape measures, levels, etc.) or with topographic methods and in any case the results obtained using these methods are undoubtedly less reliable.

However, although these techniques offer the potential for improving the working methods currently employed in most companies in the sector, they do have some drawbacks. The two major disadvantages are the cost of the equipment and the highly specialised, laborious and lengthy data processing work required to develop the 3D models. Although it is likely that equipment costs will fall and that data processing and 3D model creation will become less complex in the future, a solution for the present is to hire the equipment and to outsource the computer work to less costly countries.

\section{REFERENCES}

[1] COM 2003, 232. (Commission of the European Communities, 232) Seventh report on the situation in world shipbuilding, from http://europa.eu.int/, accessed on 8-07-2006

[2] Karunakaran, K. P.; Agrawal, S.; Vengurlekar, P. D.; Sahasrabudhe, O. S.; Pushpa, V.; Ely, R. (2005). Segmented object manufacturing, IIE Transactions, Vol. 39, 291-302

[3] Bordegoni, M.; Filippi, S. (2001). Reverse Engineering for Molding, Proceedings of ADM 2001 $12^{\text {th }}$ Intl. Conference on Design Tools and Methods in Industrial Engineering, A2, 28-37

[4] Lichti, D. D.; Gordon, S. J.; Stewar, M. P. (2002). Ground-Based Laser Scanners: operation, systems and applications, Geomatica, Vol. 56, No. 1, 21-33

[5] Gruen, A.; Akca, D. (2005). Least squares 3D surface and curve matching, ISPRS Journal of Photogrammetry and Remote Sensing, Vol. 59, No. 3, 151-174

[6] Yau, H. T.; Lee, R. K.; Chuang, C. M.; Hsu, C. Y. (2005). NC Tool-Path Generation Based on Surfel Models Constructed from Random Scanned Data, Computer-Aided Design \& Applications, Vol. 2, 1-4

[7] Floater, M. S. (1997). Parameterization and smooth approximation of surface triangulation, Computer Aided Geometric Design, Vol. 14, No. 3, 231-250

[8] Benko, P.; Martin, R. R.; Várady, T. (2001). Algorithms for reverse engineering boundary representation models, Computer Aided Design, Vol. 33, No. 11, 839-851

[9] Liu, G. H.; Wong, Y. S.; Zhang, Y. F.; Loh, H. T. (2003). Modelling cloud data for prototype manufacturing, Journal of Materials Processing Technology, Vol. 138, 53-57

[10] Leica Geosystems AG. From http://www.leica-geosystems.com, accessed on 6-11-2005

[11] Yin, Z.; Jiang, S. (2004). Iso-phote based adaptive surface fitting to digitized points and its applications in region-based tool path generation, slicing and surface triangulation, Computers in Industry, Vol. 55, 15-28

[12] Geng, J.; Zhuang, P.; May, P.; Yi, S.; Tunnell, D. (2004). 3D FaceCam - A Fast and Accurate 3D Facial Imaging Device for Biometrics Applications. In A. K. Jain \& N. K. Ratha (eds), Proceedings SPIE, Biometric Technology for Human Identification, 5404, 316-327

[13] Boehnen, C.; Flynn, P. (2005). Accuracy of 3D Scanning Technologies in Face Scanning Scenario, Proceedings of the $5^{\text {th }}$ International Conference on 3D Digital Imaging and Modelling, CD-ROM, 310-317 